\title{
Neosexismo y creencias desfavorables hacia la mujer líder
}

\author{
Alejandro Jesús Delgado Iglesias, Ana Belén Palma Navarro y Ángela Rivas Pajares. \\ Universidad de Granada
}

\begin{abstract}
Recepción: 12 de mayo de 2015 | Revisión: 17 de junio de 2015 | Aceptación/Publicación: 17 de julio de 2015
Artículo tutorizado por Antonia Calvo Salguero, profesora del Departamento de Psicología Social de la Universidad de Granada Citar: Delgado, A.J., Palma, A.B. y Rivas, A. (2015). Neosexismo y creencias desfavorables hacia la mujer líder. ReiDoCrea, 4, 117-122.
\end{abstract}

Resumen: A pesar de todos los avances que se han producido en materia de igualdad de género en nuestra sociedad, el número de mujeres en puestos directivos sigue siendo notablemente inferior en comparación con el de los varones. En el presente estudio se aborda el análisis de factores que permitan explicar esta desigual ocupación de puestos de directivos o de liderazgo en función del género. Diversos estudios han señalado que las actitudes desfavorables hacia la mujer líder constituyen un obstáculo para el desarrollo profesional de las mujeres que desean desempeñar este tipo de puestos. Aunque la literatura ha analizado diversos factores explicativos de estas actitudes, escasos estudios han analizado el posible papel explicativo que puede tener el neosexismo. Así, el objetivo principal de este estudio es comprobar si el neosexismo predice las actitudes negativas hacia la mujer en puestos directivos, cuando se controla la edad, el género y el nivel educativo. Para cubrir este objetivo se usó una muestra de 126 trabajadores/as de distintas provincias andaluzas. Se aplicó el cuestionario de neosexismo de Tougas et al. (1995) y la escala de actitudes hacia la mujer líder de Lupano y Castro (2011). Se realizaron análisis de correlaciones y de regresión lineal por bloques. Los resultados indicaron que cuanto mayor es la adherencia a las creencias neosexistas, menor es la creencia de que las mujeres posean las características masculinas asociadas al liderazgo. Se concluye que el neosexismo predice actitudes negativas hacia la mujer líder.

Palabras clave: neosexismo, crencias desfavorables hacia la mujer líder, trabajadores/as, liderazgo

\section{NEOSEXISM AND UNFAVORABLE BELIEFS TOWARD FEMALE LEADER}

\begin{abstract}
Despite the advances that have been made regarding gender equality, the number of woman in managerial positions is still notably inferior to the number of men. In this study we analyze the different factors that explain this gender inequality. Previous studies have shown that the negative and unfavorable attitudes towards female leaders create an obstacle to the professional development of woman who desire to obtain these kind of jobs. Even though there have been various studies that analyze different factors as to why these negative attitudes towards female leaders occur, few have analyzed the possibility that this is due to the neosexism. This being said, this studies main objective is to check if neosexism can predict the negative attitudes that exist toward female leaders. To do so, we will control other factors, such as: sex, age and the education level of the test subjects. For this study, we used a sample of 126 workers, all from different regions of Andalusia, Spain. We then used the Tougas et al (1995) neosexism questionnaire and the Lupano and Casrto (2011) scale of attitudes toward the female leader. To obtain the results, we did a correlational analysis and a block linear regression. The results of these questionnaires indicated that the higher the fixation with neosexism, the lower are the beliefs that woman are capable of possessing the masculine qualities that are associated to a good leader. In conclusion, neosexism does predict negative attitudes towards female leaders.
\end{abstract}

Key words: neosexism, unfavorable beliefs toward female leader, workers, leadership.

\section{Introducción}

A pesar de todos los avances que se han producido en materia de igualdad de género en nuestra sociedad, el número de mujeres en puestos directivos sigue siendo notablemente inferior en comparación con el de los varones. Así, en España, según datos del INE (2014), en 2012 las mujeres tan solo ocupaban el 13,3\% de los puestos de la presidencia y de los consejos administrativos del IBEX 35, y en 2013, frente al $8,6 \%$ de los hombres, tan solo el $4,4 \%$ de las mujeres ocupaban puestos de dirección de pequeña empresa, de departamento o de sucursal.

La literatura ha ofrecido múltiples teorías para explicar este fenómeno. No obstante, la explicación más relevante se ha basado en la existencia e influencia de los estereotipos de género (Cuadrado, 2011). Los estereotipos de género son creencias 
socialmente compartidas sobre las características de los roles que tradicionalmente han desempeñado hombres y mujeres en una sociedad. Estas creencias estereotipadas de género son con frecuencia las responsables de los prejuicios y de la discriminación de género en el contexto laboral (Eagly \& Mladinic, 1994).

En esta línea, la Teoría de la Congruencia del Rol (Eagly \& Karau (2002), presenta el prejuicio hacia las mujeres líderes como uno de los factores causales de los problemas y obstáculos que experimentan las mujeres para acceder a puestos de liderazgo. Diversos estudios encuentran que cuando las personas piensan en un líder, piensan en cualidades masculinas, fenómeno que Schein (2001) denominó "think managerthink male". Este conduce a que las personas perciban, por tanto, a los hombres como más competentes, capaces y eficaces que las mujeres para ejercer el liderazgo. El prejuicio se generaría a partir de la incongruencia entre las cualidades masculinas que se asocian al rol del líder y las características femeninas asociadas a las mujeres. Por lo que, se percibe que las mujeres no poseen las cualidades y capacidades necesarias para desempeñar dichos puestos de manera eficaz.

Numerosos estudios realizados en diversas culturas ponen de manifiesto que son los hombres quienes mayoritariamente mantienen estas creencias y que se trata de un fenómeno que, ocurre en múltiples y diversas culturas del mundo (Cuadrado, GarcíaAel, \& Molero, 2007). Dado que los altos cargos directivos están dominados por varones, estas creencias desfavorables respecto a las competencias de la mujer para ocupar puestos de liderazgo, tienen un papel clave en el desarrollo de la carrera profesional de las mujeres que desean desempeñar esos puestos. Desde esta perspectiva, el análisis de los factores que generan estas creencias cobra especial relevancia, ya que su conocimiento permitiría elaborar programas de intervención que condujeran al cambio de creencias desfavorables por otras más favorables.

En este contexto, el sexismo emerge como un posible factor determinante de las mismas. El sexismo puede ser entendido como una actitud que, atendiendo a los estereotipos que socialmente se asignan a hombres y mujeres, valora a las personas en función de su sexo biológico (Expósito, Moya, \& Glick, 1998). Actualmente se distingue entre el "viejo sexismo" y el "nuevo sexismo". El primero puede ser definido como una actitud de prejuicio basada en la supuesta inferioridad o diferencia de las mujeres como grupo (Glick \& Fiske, 1996). El nuevo sexismo o neosexismo es una nueva forma de entender y de manifestar el viejo sexismo. Este enfoque se caracteriza por negar la existencia de discriminación hacia las mujeres. El concepto pretende reflejar un prejuicio encubierto y sutil acerca de la valoración negativa del género femenino, no siendo éste tan visible como en el viejo sexismo (Goodwin \& Fiske, 2001), pero que sigue contribuyendo al mantenimiento de las desigualdades de género (Barreto \& Ellemers, 2005).

El neosexismo ha sido definido por Tougas, Brown, Beaton, \& Joly (1995) como "manifestación de un conflicto entre los valores igualitarios y sentimientos negativos residuales hacia las mujeres" (p. 843). Esta concepción del nuevo sexismo se centra en la esfera laboral. Algunos estudios han puesto de manifiesto que el viejo sexismo y/o el "sexismo hostil" se relaciona con creencias y actitudes negativas hacia la mujer directiva (Masser \& Abrams, 2004; Sakalli-Ugurlu \& Beydogan, 2002). Sin embargo, los autores de este estudio no han logrado encontrar en la revisión de la literatura estudios que hayan analizado la influencia del neosexismo en la esfera laboral, tal como fue concebido por Tougas et al. (1995), sobre estas creencias. Por ello, el objetivo principal de este estudio es comprobar si el neosexismo predice las creencias desfavorables hacia las competencias de las mujeres para realizar puestos directivos. 
Tomando como marco de referencia la literatura revisada, se estableció la siguiente hipótesis:

Hipótesis: El nivel de neosexismo predice en qué medida las personas consideran que las mujeres presentan características masculinas comúnmente asociadas al liderazgo, de tal manera que cuanto mayor sea el nivel de neosexismo, menor es la creencia de que las mujeres presenten estas características y, por tanto, más negativa es la actitud hacia la mujer en puestos directivos.

\section{Control de variables}

Dado que la edad, el nivel educativo y el género han sido relacionadas en diversos estudios con el sexismo y las creencia y actitudes hacia las mujeres directivas (Eagly, Makhijani, \& Klonsky, 1992; Glick, Lameiras, \& Rodríguez, 2002; Goodwin, Operario, \& Fiske, 1998; Morales \& Cuadrado, 2004; Moya \& Expósito, 2001) se controlarán sus efectos en la puesta a prueba de estas hipótesis.

\section{Metodología}

\section{Participantes y procedimiento}

Para llevar a cabo nuestra investigación se usó una muestra de 125 trabajadores (63 hombres y 62 mujeres) de diversos puestos de trabajo (por ejemplo, profesor/a, camarero/a, administrativo/a, abogado/a, cocinero/a, peluquero/a, carnicero/a, mecánico, fisioterapeuta, agricultor, contable, economista, fontanero, educador infantil, seguridad privada, soldador, gerente, enfermero, economista, etc.) de distintas provincias andaluzas. Los hombres tenían una edad media de 42.18 años (DT = 13.79) y las mujeres de 40.27 años $(D T=13.60)$. El porcentaje de participantes por nivel educativo fue el siguiente: a) Estudios primarios: $6.3 \%$; b) Graduado escolar: $21.4 \%$; c) Bachillerato o FP grado medio: $29.4 \%$; d) Diplomatura o FP Grado Superior: 19.8\%; e) Licenciatura: $17.5 \%$; d) Máster o Doctorado: 5.6\%.

La participación fue anónima y voluntaria. Los cuestionarios fueron aplicados por alumnos/as de $3^{\circ}$ grado de Psicología de la Universidad de Granada. Cada alumno/a invitó a participar en el estudio a un hombre y a una mujer trabajador/a. Una vez que los participantes entregaban los cuestionarios cumplimentados, se les agradecía el esfuerzo y el tiempo empleado en las pruebas, y en caso de estar interesado en los resultados, se les informaba de cómo y dónde podían localizarlos.

\section{Diseño}

En nuestro estudio se ha utilizado una muestra incidental y se ha efectuado un diseño correlacional en el que se han considerado los siguientes constructos: neosexismo y actitudes negativas hacia mujeres directivas, así como variables sociodemográficas tales como el sexo, la edad, el nivel de educación.

\section{Instrumentos de medida}

Para evaluar el neosexismo se usó la Escala de Neosexismo (Tougas, Brown, Beaton, \& Joly, 1995). Esta prueba fue adaptada al español por Moya y Expósito (2001). El instrumento consta de 11 ítems, con un formato de respuesta tipo Likert que engloba 7 puntos (desde 1 -Muy en Desacuerdo- hasta 7 -Muy de Acuerdo). Para su interpretación, debemos invertir los ítems 2 y 11. Las puntuaciones más altas se corresponden con un talante más neosexista. Un ejemplo de ítem es: "Las demandas 
de las mujeres en términos de igualdad entre los sexos son simplemente exageradas". El coeficiente alfa obtenido en este estudio fue .71.

Para evaluar las actitudes negativas hacia las mujeres en puestos de dirección se usó la sub-escala que evalúa la Dimensión Instrumental de la Escala de actitudes desfavorables hacia mujeres líderes "ACT-ML" (Lupano \& Castro, 2011). Esta subescala evalúa en qué medida las personas consideran que las mujeres presentan características masculinas asociadas al liderazgo. Esta sub-escala contiene 3 ítems a los que se debe responder en una escala de 1 a 7 puntos (desde 1 -Muy en Desacuerdo- hasta 7 -Muy de Acuerdo-). A mayor puntuación en esta escala, menor es la creencia de que las mujeres presenten características masculinas asociadas al liderazgo, y por consecuente, la actitud que se muestre hacia las mujeres directivas será más reacia o negativa. Un ejemplo de ítem es: "Las mujeres no son lo suficientemente ambiciosas como para ser exitosas en el mundo laboral". El coeficiente alfa obtenido en este estudio fue .88.

\section{Resultados}

Los diferentes análisis estadísticos realizados fueron: correlaciones de Pearson y análisis de regresión por bloques. Se usó el paquete estadístico SPSS 20. Los estadísticos descriptivos pueden observarse en la Tabla 1.

\begin{tabular}{|lcc|}
\hline \multicolumn{3}{|c|}{ Tabla 1 } \\
\hline \multicolumn{3}{|c|}{ Estadísticos descriptivos } \\
\hline D. INSTRUMENTAL & 1,776 & Desviación típica \\
EDAD & 41,216 & 13,674 \\
EDUCACION & 3,392 & 1,307 \\
SEXO & 1,504 &, 502 \\
NEOSEXISMO & 2,271 &, 743 \\
\hline
\end{tabular}

Los resultados de los análisis de regresión indicaron que en el primer bloque, cuando se controlaron las variables demográficas, el sexo predijo la dimensión instrumental ( $\beta$ $=-.310 ; p=.001)$. No obstante, cuando se introduce el neosexismo como variable predictora, sólo el neosexismo, predijo significativamente la dimensión instrumental ( $\beta$ $=.439 ; p=.001$ ). La varianza explicada por las variables controladas (edad, sexo y nivel educativo) en el primer bloque fue de $1.28 \%$. No obstante, la varianza explicada cuando se introduce la variable predictora neosexismo en el segundo bloque fue de $2.52 \%$. El cambio de $\mathrm{R}^{2}$ fue significativo $(p=.000)$. Los resultados del análisis de correlaciones pueden observarse en la Tabla 2.

\begin{tabular}{|c|c|c|c|c|}
\hline \multicolumn{5}{|c|}{$\begin{array}{c}\text { Tabla } 2 \\
\text { Correlaciones }\end{array}$} \\
\hline & EDAD & SEXO & EDUCACIÓN & NEOSEXISMO \\
\hline EDAD & - & & & \\
\hline SEXO & -.070 & - & & \\
\hline EDUCACIÓN & -.094 & $.236^{\star *}$ & - & \\
\hline NEOSEXISMO & -.065 & $-.581^{* *}$ & $-.227^{*}$ & - \\
\hline D.INSTRUMENTAL & .013 & $-.330^{* *}$ & $-.181^{*}$ & $.481^{* *}$ \\
\hline${ }^{* *} p<.005 ;{ }^{*} p<.05$ & & & & \\
\hline
\end{tabular}




\section{Discusión y conclusiones}

El objetivo principal del presente estudio fue comprobar si el neosexismo predice las creencias desfavorables sobre las competencias de las mujeres para ocupar puestos directivos. Tomando como marco de referencia la literatura revisada, se estableció una hipótesis en la que se proponía que el nivel de neosexismo predice en qué medida las personas consideran que las mujeres presentan características masculinas comúnmente asociadas al liderazgo, de tal manera que cuanto mayor sea el nivel de neosexismo, menor es la creencia de que las mujeres presenten características masculinas asociadas al liderazgo.

Los resultados pusieron de manifiesto que el neosexismo se relaciona y predice la Dimensión Instrumental. Por tanto, los resultados apoyan la hipótesis establecida. Estudios previos han puesto de manifiesto cómo el viejo sexismo y/o el "sexismo hostil" se relaciona con creencias y actitudes negativas hacia la mujer directiva (Masser \& Abrams, 2004; Sakalli-Ugurlu \& Beydogan, 2002). En estos estudios también se analizaron el valor predictivo del sexismo benevolente, entendido éste, al igual que el neoseximo, como una nueva forma de expresar creencias sexistas. Sin embargo, este tipo de sexismo no predijo en estos estudios las creencias y/o actitudes hacia la mujer líder. Creemos que en estos estudios no se obtuvieron relaciones significativas debido a que, tal como sugieren Glick, Diebold, Bayle-Werner, \& Zhu (1997), el sexismo benevolente va dirigido a las mujeres tradicionales o no profesionales y tiene un tono afectivo positivo, representando una recompensa para este tipo de mujeres, ya que éstas aceptan la supremacía masculina; mientras que el sexismo hostil va dirigido a las mujeres profesionales o no tradicionales y tiene un tono afectivo negativo, representando un castigo para este tipo de mujeres, ya que éstas no aceptan los roles tradicionales. Por lo que, el sexismo benevolente, al ir dirigido hacia las mujeres tradicionales o no profesionales, puede perder capacidad predictiva respecto a las mujeres profesionales o en contextos laborales. Dado que el neosexismo, tal como lo evalúa la escala de Tougas, Brown, Beaton, \& Joly (1995), va dirigido a captar las nuevas creencias sexistas en la esfera laboral, éste puede capturar mejor la relación entre las nuevas creencias sexistas y las creencias o actitudes hacia la mujer líder.

Cabe señalar que una de las limitaciones de este estudio fue el uso de la escala de actitudes hacia la mujer líder que, aunque traducida en lengua castellana y con un buen coeficiente alfa en este estudio, se validó en una muestra argentina. Por lo que, futuras investigaciones con muestras españolas deberían validar previamente la escala en el contexto español.

A modo de conclusión, puede decirse que los resultados apoyan la idea de que el neosexismo predice las creencias de que las mujeres no poseen las características masculinas que se asocian al liderazgo y, por tanto, predice actitudes más negativas hacia la mujer líder. En consecuencia, podría afirmarse, de acuerdo con la Teoría de la Congruencia de Rol, que los prejuicios sexistas respecto al papel de las mujeres en el mundo laboral conducen a percibir a las mujeres como menos capaces y competentes para ejercer el liderazgo.

\section{Referencias}

Barreto, M. \& Ellemers, N. (2005). The burden of benevolent sexism: How it contributes to the maintenance of gender inequalities. European Journal of Social Psychology, 35, 633-642. 
Cuadrado, I., García-Ael, C., \& Molero, E. (2007). Género y dirección: un análisis del estereotipo "think-manager - think male". En C. L. Guillén y R. Guil (Coords.), Psicología Social: un encuentro de perspectivas (pp. 1410-1413). Cádiz: Asociación de Profesionales de la Psicología.

Eagly, A.H. \& Karau, S.J. (2002). Role congruity theory of prejudice toward female leaders. Psychological Review, 109, 573-598.

Eagly, A.H. \& Mladinic, A. (1994). Are people prejudiced against women? Some answers from research on attitudes, gender stereotypes, and judgments of competence. European Review of Social Psychology, 5, 1-35.

Eagly, A.H. \& Carli, L.L. (2007). Through labyrinth: truth about how women become leaders. Boston: Harvard University Bussiness School Press.

Eagly, A.H., Makhijani, M.G. \& Klonsky, B.G. (1992). Gender and the evaluation of leaders: A Meta-analysis. Psychological Bulletin, 111, 3-22.

Expósito, F., Moya, M., \& Glick, P. (1998). Sexismo ambivalente: medición y correlatos. Revista de Psicología Social, 2, 159-169.

Glick, P.; Diebold, J., Bayle-Werner, B. \& Zhu, L. (1997). The two faces of Adam: Ambivalent sexism and polarized attitudes toward women. Personality and Social Psychology Bulletin, 23, 1323-1334.

Glick, P. \& Fiske, S.T. (1996). The ambivalent sexism inventory: differentiating hostile and benevolent sexism. Journal of Personality and Social Psychology, 70, 491-512.

Glick, P., Lameiras, M., \& Rodríguez, Y. (2002). Education and catholic religiosity as predictors of hostile and benevolent sexism toward women and men. Sex Roles, 47, 433-441.

Goodwin, S.A. \& Fiske, S.T. (2001). Power and gender: The double-edged sword of ambivalence. En R. K. Unger (Ed.), Handbook of the psychology of women and gender (pp. 358-366). New York: Wiley.

Goodwin, S.A., Operario, D., \& Fiske, S.T. (1998). Situational power and interpersonal dominance facilitate bias and inequality. Journal of Social Issues, 54, 677-698.

INE (Instituto Nacional de Estadística) (2014). Mujeres y Hombres en España. Catálogo de publicaciones de la Administración General del Estado. http://publicacionesoficiales.boe.es

Lupano-Perugini, M.L. \& Castro-Solano, A. (2011). Unfavorable attitudes towards women leaders. An instrument to assess them. Summa Psicologica, 8, 19-29.

Masser, B.M. \& Abrams, D. (2004). Reinforcing the glass ceiling: the consequences of hostile sexism for female managerial candidates. Sex Roles, 51, 609-615.

Morales, J. F. \& Cuadrado, I. (2004). Introducción: Teoría de congruencia de rol del prejuicio hacia líderes femeninos. Revista de Psicología General y Aplicada, 57, 135- 146.

Moya, M., \& Expósito, F. (2001). Nuevas formas, viejos intereses: neosexismo en varones españoles. Psicothema, 13, 643-649.

Sakallı-Uğurlu, N., \& Beydoğan, B. (2002). Turkish college students' attitudes toward women managers: the effects of patriarchy, sexism, and gender differences. The Journal of Psychology, 136, 647-656.

Schein, V.E. (2001). A global look at psychological barriers to women's progress in management. Journal of Social Issues, 57, 675-688.

Tougas, F., Brown, R., Beaton, A.M., \& Joly, S. (1995). Neosexism: Plus Ça Change, Plus Cést Pareil. Personality and Social Psychology Bulletin, 21, 842-849. 